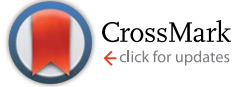

Cite this: RSC Adv., 2017, 7, 9620

Received 9th January 2017 Accepted 23rd January 2017

DOI: 10.1039/c7ra00305f

rsc.li/rsc-advances

\section{Furan-carboxamide derivatives as novel inhibitors of lethal H5N1 influenza A viruses $\uparrow$}

\begin{abstract}
Yongshi Yu, ${ }^{a}$ Jie Zheng, ${ }^{a}$ Lei Cao, ${ }^{\text {b }}$ Shu Li, ${ }^{c}$ Xiwang Li, ${ }^{a}$ Hai-Bing Zhou, ${ }^{a}$ Xianjun Liu, ${ }^{d}$ Shuwen $\mathrm{Wu}^{\star \mathrm{b}}$ and Chune Dong ${ }^{\star \mathrm{a}}$

In this study, we reported the synthesis and biological characterization of a novel series of furancarboxamide derivatives that were potent inhibitors of the influenza A H5N1 virus. The systematic structure-activity relationship (SAR) studies demonstrated that the 2,5-dimethyl-substituted heterocyclic moiety (furan or thiophene) had significant influence on the anti-influenza activity. In particular, 2,5dimethyl-N-(2-((4-nitrobenzyl)thio)ethyl)-furan-3-carboxamide 1a showed the best activity against the $\mathrm{H} 5 \mathrm{~N} 1$ virus with an $\mathrm{EC}_{50}$ value of $1.25 \mu \mathrm{M}$. For the first time, the simple scaffold furan-carboxamide derivatives were identified as novel inhibitors of lethal H5N1 influenza A virus.
\end{abstract}

Influenza is an acute respiratory infectious disease, caused by influenza viruses. Due to the antigenic drift and antigenic shift, influenza viruses can result in periodic epidemics with high morbidity and mortality. ${ }^{1,2}$ Influenza A virus subtype H5N1 is a virulent influenza in birds and is highly pathogenic, which has made the likelihood of human influenza pandemic and the possible socioeconomic impact of major worldwide concern. $^{3-6}$ Since 2003, the HPAI H5N1 strains have led to several hundred deaths, and has much higher mortality, about $60 \%$ compared with other influenza strains. ${ }^{7-9}$ Recently, several research groups have reported that HPAI H5N1 virus could attain aerosol transmissibility in mammalians by acquiring HPAI $\alpha$-2, 6-linked sialic acid (human-type receptor) binding ability or by resorting with the $2009 \mathrm{H} 1 \mathrm{~N} 1$ pandemic

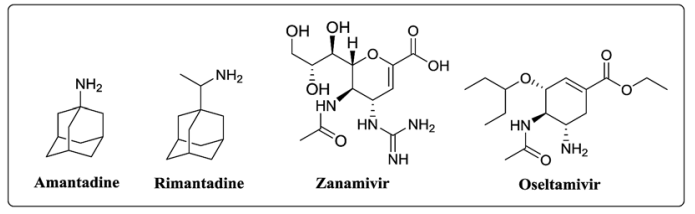

Fig. 1 Approved drugs for influenza infection.

${ }^{a}$ Hubei Provincial Key Laboratory of Developmentally Originated Disease, Hubei Province Engineering and Technology Research Center for Fluorinated Pharmaceuticals, Wuhan University School of Pharmaceutical Sciences, Wuhan 430071, China. E-mail: cdong@whu.edu.cn

${ }^{b}$ State Key Laboratory of Virology, College of Life Sciences, Wuhan University, Wuhan 430072, China. E-mail: shuwenwu@hotmail.com

'Medical Research Institute, Wuhan University, Wuhan 430071, China

${ }^{d}$ Suzhou ChukaipharmaTech, Building 14, 2358 Changan Road, Wujiang 215200, Suzhou, China

$\dagger$ Electronic supplementary information (ESI) available: NMR spectra of final compounds 1a-1v. See DOI: 10.1039/c7ra00305f virus. ${ }^{11}$ Therefore, its potential influence on ecology and human health is becoming more and more worthy of consideration. ${ }^{10}$ Currently, there are two classes of anti-influenza drugs for the interruption of specific processes in influenza infection, which are neuraminidase inhibitors, like zanamivir and oseltamivir and inhibitors of the viral M2 protein, such as amantadine (AMD) and rimantadine (Fig. 1). However, the emergence of drug-resistant influenza viruses has limited the use of these drugs, making the identification of novel anti-

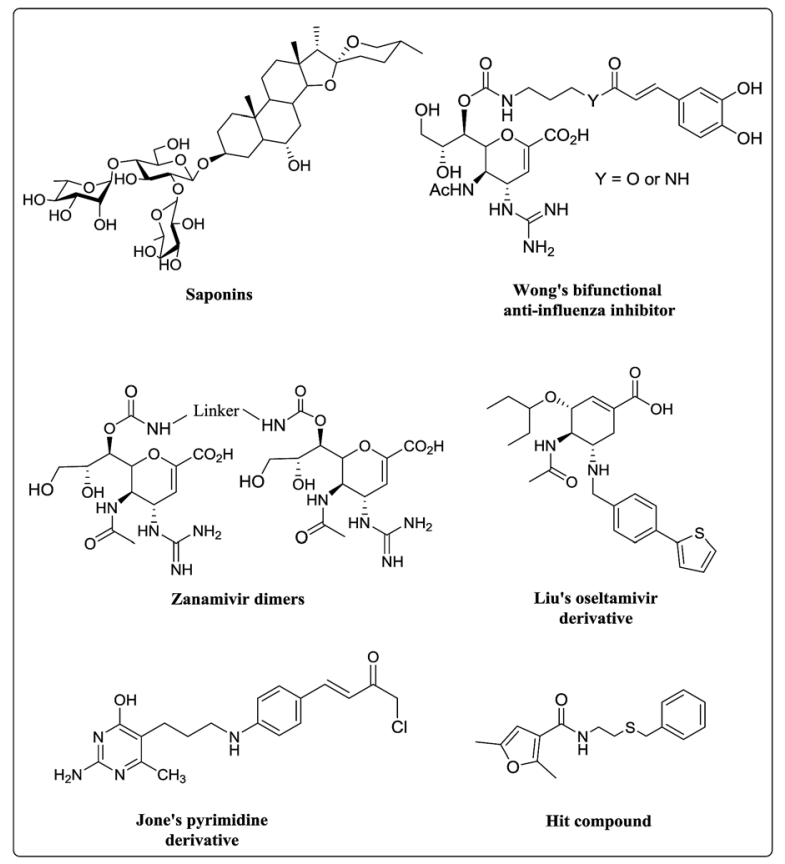

Fig. 2 Structures of known small molecular inhibitors of H5N1. 
influenza drugs an urgent task. ${ }^{\mathbf{1 1}}$ Therefore, efforts to develop improved and new antiviral drugs will be crucial for controlling future influenza outbreak.

Consequently, many researchers have expended considerable efforts in the pursuit of antiviral small molecules via bioinformatics studies, hit-and-lead discovery approaches and structural modifications of approved anti-influenza drugs (Fig. 2). ${ }^{12}$ In 2005 Tucker reported the zanamivir (ZMV) dimers were highly potent neuraminidase inhibitors against H5N1 virus and compared with $\mathrm{ZMV}$, high concentration of dimers was found in rat lung tissue after 1 week. ${ }^{13}$ Jones and coworker utilized computational molecular docking identifying 4-(4-((3(2-amino-4-hydroxy-6-methyl-5-pyrimidinyl)propyl)-amino) phenyl)-1-chloro-3-buten-2-one, which could inhibit both H1N1 and $\mathrm{H} 5 \mathrm{~N} 1$ viruses. Comparable to the known neuraminidase inhibitor oseltamivir, this compound remained effective in the presence of virus mutations conferring resistance to either oseltamivir or zanamivir and also vice versa ${ }^{\mathbf{1 4}}$ Almost at the same time, Li's group reported the first small molecule inhibitors saponins with 3-O- $\beta$-chacotriosyl residue, which showed potent inhibitory activity with $\mathrm{IC}_{50}$ of 7.22-9.25 $\mu \mathrm{M}$. The subsequent SAR studies showed the 3-O- $\beta$-chacotriosyl residue was essential for the activity, and the aglycone structure also affected the activity. ${ }^{15}$ In 2014 Liu et al. discovered that two series of oseltamivir derivatives were the most potent N1-selective inhibitors, with $\mathrm{IC}_{50}$ values of $0.0019,0.0038$, and $0.0067 \mu \mathrm{M}$ against NAs from three H5N1 viruses, respectively. Molecular docking studies revealed that the 150-cavity was an auxiliary binding site that may contribute to the high selectivity of these compounds. ${ }^{16}$ Wong and coworkers explored the novel dual-targeted bifunctional antiinfluenza drugs formed by conjugation zanamivir with antiinflammatory agents, in which, the caffeic acid bearing zanamivir conjugates showed simultaneous inhibition of influenza virus neuraminidase and suppression of pro-inflammatory cytokines. These conjugates provided remarkable protection of the lethally infected mice by H1N1 and H5N1 influenza viruses. ${ }^{17}$

As a part of our long-term interest in development of antiviral agents, ${ }^{\mathbf{1 8 - 2 1}}$ as well as further exploring anti-influenza drugs that might have superior efficacy and fewer side effects than existing therapeutic agents, the furan-carboxamide derivatives were then identified with significant antiviral activity based on the result of our previous work. The hit compound 2,5-dimethyl- $N$-(2-(benzyl) thio)ethyl)furan-3-carboxamide (Fig. 2) based on a furan scaffold showed micromolar potency against the H5N1 virus. The subsequent chemical optimization led to potential compound 1a with improved bioactive properties and higher selectivity index. It is worth noting that these compounds are simple, particularly, as far as we are known, there is no furan-carboxamide scaffold has been reported as an $\mathrm{H} 5 \mathrm{~N} 1$ inhibitor. Hence, an attempt has been made to explore the potential of these newly discovered carboxamides as leads for anti-influenza drug development.

\section{Results and discussion}

\section{Chemistry}

The general approach for the synthesis of the furancarboxamide derivatives was outlined in Scheme 1. First, the starting materials amine $\mathbf{2}$ and carboxylic acid $\mathbf{3}$ were all synthesized according to previous reported methods. ${ }^{22-26}$ With all these compounds in hand, the target products were obtained in one step through the simple coupling reaction of 2 and 3 to give 1 in $30-80 \%$ yields.

\section{Structure-activity relationship analysis}

The activity of all new carboxamide compounds were tested against $\mathrm{H} 5 \mathrm{~N} 1$ virus. Amantadine was included in all experiments as the reference compound. The results of this extensive biological evaluation are shown in Table 1. As a global observation, it is noteworthy that the substituents on the phenyl ring play important roles in the activity. Initially, $\mathrm{N}$-(2-(benzylthio) ethyl)-2,5-dimethylfuran-3-carboxamide $\mathbf{1 b}$ showed good activity against influenza $\mathrm{H} 5 \mathrm{~N} 1$ virus $\left(\mathrm{EC}_{50}=7.716 \mu \mathrm{M}\right.$, entry 2$)$. Then the diverse functional groups, such as fluoro, bromo, chloro, nitro and trifluoromethyl etc. were introduced at the 4position of the phenyl ring (1a and 1c-1i). Fortunately, we found compound 1a with 4-nitro group showed potent activity with $\mathrm{EC}_{50}$ value $1.25 \mu \mathrm{M}$ compared to $\mathrm{AMD}\left(\mathrm{EC}_{50}=0.551 \mu \mathrm{M}\right.$, entry 1). Moreover, the compounds substituted with electron withdrawing groups at the phenyl ring were more potent in the cellular assay than the derivatives with electron donating groups (entries 2-9). Among them, $N$-(2-((4-cyanobenzyl)thio)ethyl)-2,5-dimethylfuran-3-carboxamide 1f showed the good cellular potency $\left(\mathrm{EC}_{50}=5.302 \mu \mathrm{M}\right.$, entry 6). For instance, analogues 14 and 15 containing the methyl group and the methoxyl group respectively, exhibited poor anti-influenza activity. Surprisingly, when the nitro group was introduced at the 2-position of the phenyl ring, no inhibition was observed (entry 10). While, the compound $\mathbf{1 k}$, in which the nitro group was introduced at the 3-position of the phenyl, exhibited slightly decreased potency with $\mathrm{EC}_{50}$ values $9.970 \mu \mathrm{M}$ (entry 11). We next attempted to replace the furan ring of $1 \mathrm{a}$ with a thiophene ring. Fortunately, in most cases, the thiophene compounds displayed more potent in the cellular assay than their furan derivatives (entries 16 and 19). Compound $\mathrm{N}$-(2-((4cyanobenzyl)thio)-ethyl)-2,5-dimethylthiophene-3-carboxamide 1t showed the higher cellular potency with $\mathrm{EC}_{50}$ value $1.338 \mu \mathrm{M}$ (entry 20). Moreover, compound $\mathbf{1 q}$ and 1r, in which the thiazole or phenyl ring was introduced, no inhibition was observed, suggesting that the thiophene or furan ring was the main contributor for the inhibition activity (entries 17 and 18). Unfortunately, as for analogues 1c to 1e, replacing the 4-nitro substituent with halogen substituents, the potency was decreased more than 7 folds (entries 3 to 5). Moreover, probably

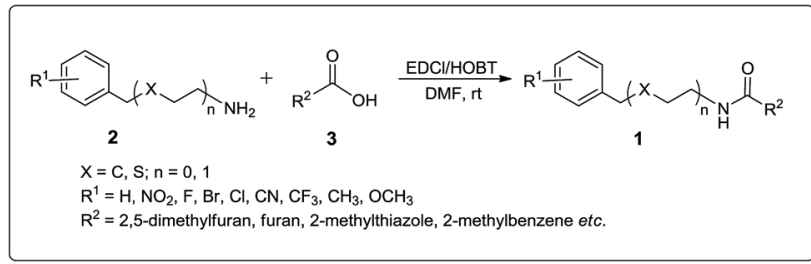

Scheme 1 Synthesis of furan-carboxamide derivatives. 
Table 1 Structure and activity of furoamide-based derivatives and their analogues $1^{a}$

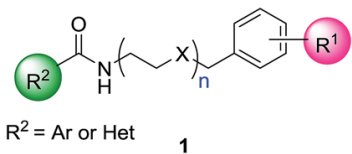

Entry

1<smiles>Cc1cc(C(=O)NCSCc2ccc([N+](=O)[O-])cc2)c(C)o1</smiles><smiles>Cc1cc(C(=O)NCSc2ccccc2)c(C)o1</smiles><smiles>Cc1cc(C(=O)NCCSc2ccc(F)cc2)c(C)o1</smiles><smiles>Cc1cc(C(=O)NCCc2ccc(Cl)cc2)c(C)o1</smiles><smiles>Cc1cc(C(=O)NCCc2ccc(Br)cc2)c(C)o1</smiles><smiles>Cc1cc(C(=O)NCCc2ccc(N)cc2)c(C)o1</smiles><smiles>Cc1cc(C(=O)NCCSc2ccc(C(F)(F)F)cc2)c(C)o1</smiles>
$1 \mathrm{~h}$<smiles>Cc1ccc(CSCNC(=O)c2cc(C)oc2C)cc1</smiles><smiles>COc1ccc(CSCNC(=O)c2cc(C)oc2C)cc1</smiles>
$1 i$<smiles>Cc1cc(C(=O)NCCSc2ccccc2[N+](=O)[O-])c(C)o1</smiles>

$\mathbf{k}$<smiles>Cc1cc(C(=O)NCCSc2cccc([N+](=O)[O-])c2)c(C)o1</smiles><smiles>Cc1cc(C(=O)NCCSc2cccc(Cl)c2)c(C)o1</smiles><smiles>O=C(NCSCc1ccc(Cl)cc1)c1ccoc1</smiles>

14

$$
\mathrm{EC}_{50}{ }^{b}(\mu \mathrm{M})
$$

$1.25 \pm 0.33$

$7.72 \pm 2.9$

$13.77 \pm 5.10$

$8.54 \pm 3.15$

$15.11 \pm 4.75$

$5.30 \pm 1.45$

$44.76 \pm 11.70$

$27.46 \pm 8.48$

$34.43 \pm 10.80$

$>100$

$>2.9$

$\mathrm{NA}^{e}$

$9.97 \pm 2.55$

$54.54 \pm 16.44$

$56.34 \pm 15.87$

$31.55 \pm 9.28$

$\mathrm{CC}_{50}{ }^{c}(\mu \mathrm{M})$

14.9

6.3

6.0

$51.48 \pm 10.56$

$94.03 \pm 19.71$

6.2

$>18.9$

2.5

$>3.6$

$91.75 \pm 21.62$

$109.66 \pm 27.42$

11.0
$>100$

$>1.8$

$68.19 \pm 21.10$
0.9

2.2
$\mathrm{SI}^{d}\left(\mathrm{CC}_{50} / \mathrm{EC}_{50}\right)$

$>80.0$<smiles>O=C(NCCSc1ccc(Cl)cc1)c1ccco1</smiles> 
Table 1 (Contd.)

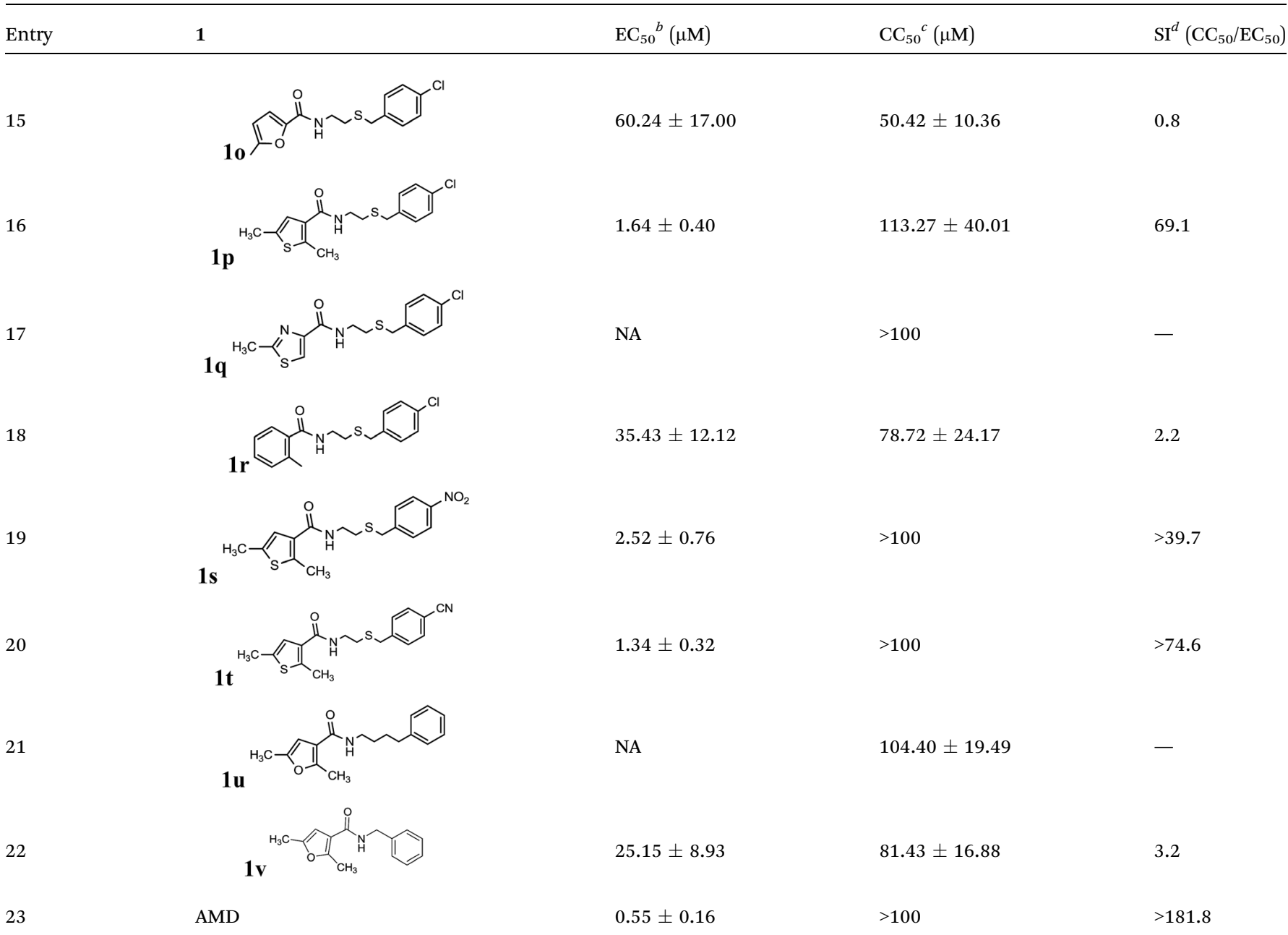

${ }^{a}$ All data are obtained for at least three independent experiments. ${ }^{b} \mathrm{EC}_{50}$, concentration that effectively inhibited the virus plaque formation by $50 \%$. ${ }^{c} \mathrm{CC}_{50}$, concentration that inhibited the MDCK cell growth by $50 \%$ compared with control cultures. ${ }^{d}$ Selectivity Index (SI) was determined for the effective compounds dividing $\mathrm{CC}_{50}$ by $\mathrm{EC}_{50} \cdot{ }^{e} \mathrm{NA}$ : no activity $\left(\mathrm{EC}_{50}>100 \mu \mathrm{M}\right) .{ }^{f}-$ : no selectivity index.

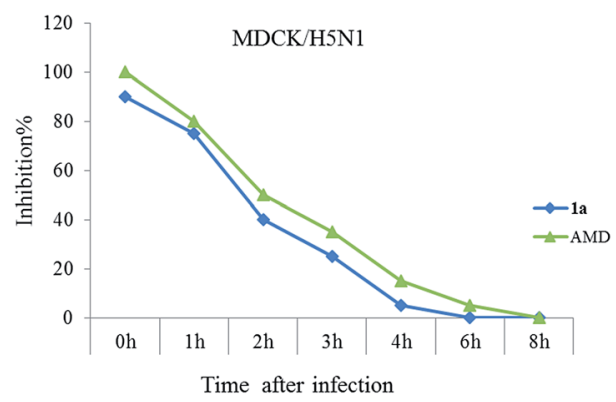

Fig. 3 Time course experiment. Compound $1 \mathrm{a}\left(c=2.1 \mu \mathrm{g} \mathrm{mL}{ }^{-1}\right)$ and AMD (amantadine, $c=0.4 \mu \mathrm{g} \mathrm{mL}^{-1}$ ) were added to the MDCK cells after being infected with $\mathrm{H} 5 \mathrm{~N} 1$ virus at an $\mathrm{MOI}$ of 2 at the indicated time.

due to the strongest electron withdrawing property of trifluoromethyl group, the activity against H5N1 virus was lost about 40 folds (entry 7). However, when changing the 4-nitro group to 4-cyano group, it displayed no significant influence on the activity (entry 6). Accordingly, the electron donating groups were also introduced in the $\mathrm{R}^{1}$ position of the phenyl ring. For instance, analogues $1 \mathrm{~h}$ and $1 \mathbf{i}$ containing the methyl group and the methoxyl group at the 4-position of phenyl ring showed inferior inhibitory activity against H5N1 virus, respectively (entries 8 and 9). In addition, decreasing the lipophilicity of derivative 1a by removing the methyl substituent on the furan ring led to inferior potency in the cell assay (entries 13-15). To further understand the roles of the linker and sulfur atom in the anti-influenza activity, analogues 2,5-dimethyl- $\mathrm{N}$-(4phenylbutyl)furan-3-carboxamide $\mathbf{1 u}$ and 2,5-dimethyl- $\mathrm{N}$-(4phenylbutyl)furan-3-carboxamide $1 \mathbf{v}$ were also synthesized for comparison. As shown in Table 1, these compounds were less potent or no inhibition compared with 1a, revealing that the linker and sulfur atom played significant roles in the inhibition (entries 21 and 22).

Anti-proliferative activities of this series compounds against MDCK cells were also evaluated to monitor the potential cytotoxic effects. As expected, none of the furan-carboxamide 

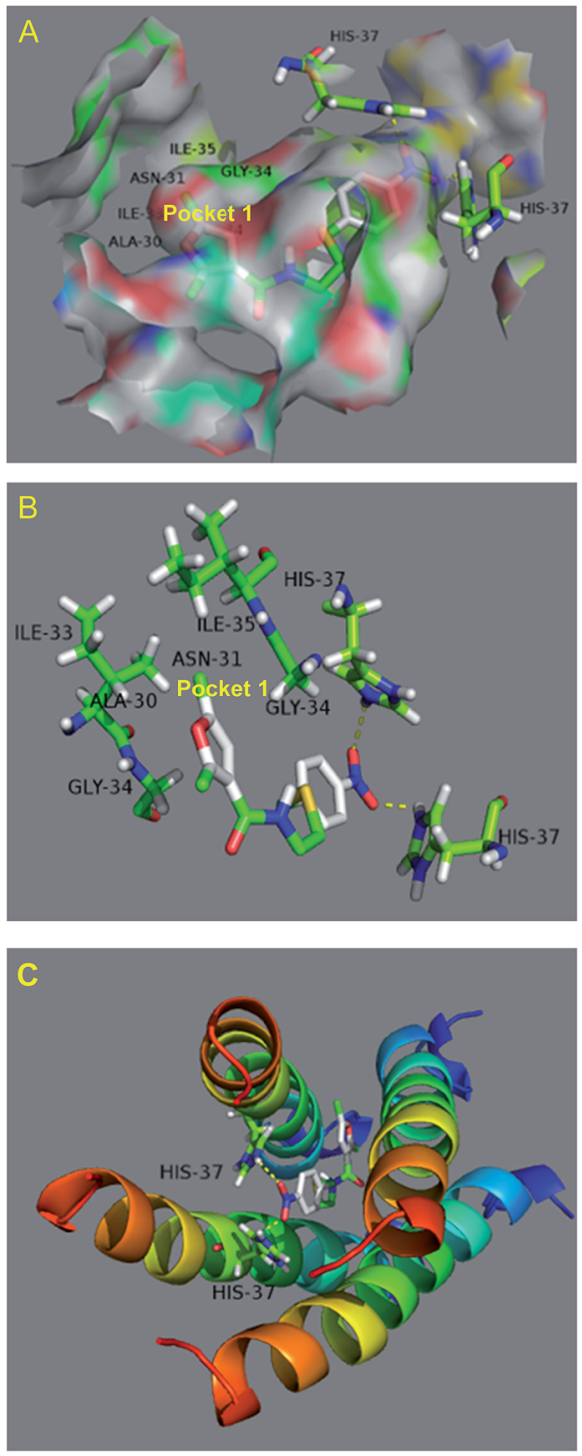

Fig. 4 (A) The surface type between 1a with M2 ion channel protein (the oxygen atoms are shown in red, the sulphur atom is shown in yellowish orange, and nitrogen atoms are shown in blue). (B) The hydrophobic interaction and hydrogen bond interactions formed between $1 \mathrm{a}$ with the backbone residues of $\mathrm{M} 2$ ion channel protein. (C) Computer modeling of the complex structures of M2 ion channel protein with 1a based on the published structure (PDB 2LYO) of M2 ion channel protein with $\mathrm{A} 2 \mathrm{Y}$.

analogues showed obvious cellular growth inhibition against MDCK cells under $50 \mu \mathrm{M}$, consolidating the anti-influenza activity of this series compounds (Table 1 ).

Preliminary mechanistic studies indicated that this series compounds inhibited the virus replication at early stages by testing compound 1a and reference agent amantadine through a time-course experiment (Fig. 3). Similar to M2 ion inhibitor amantadine, it was likely that compound 1a blocked channel function to prevent the uncoating of the viral RNPs. ${ }^{27}$

To further understand the structure-binding relationships of furan-carboxamide derivatives, the binding mode of 1a was studied by docking experiments into the M2 protein channel of influenza A virus. The docking experiments were carried out based on the published crystal structure of influenza A virus M2 protein channel (PDB 2LY0) ${ }^{28}$ with A2Y. AutoDockTool (ADT) 4.2 (ref. 29) was chosen to study the binding modes of 1a and the results showed a good binding score $\left(-5.19 \mathrm{kcal} \mathrm{mol}^{-1}\right)$. As shown in Fig. 4A, 2,5-dimethylfuran moiety of compound 1a enters into M2 protein pocket 1 formed by residues Ala-30, Asn31, Ile-33, Ile-35 and Gly-34, consolidating that the 2,5-dimethylfuran moiety played a significant role in the anti-influenza activity. Futhermore, the two oxygen atoms of nitro group of 1a form two hydrogen bonds with two backbone residues His-37 respectively (Fig. 4B and C), which was consistent with the SAR results. Further mechanism investigation is still going on in our laboratory.

\section{Conclusion}

In summary, we have synthesized a series of furan-carboxamide analogues and evaluated their biological activities against H5N1 virus. Some compounds show excellent anti-H5N1 activity and very low cytotoxicity in MDCK cells. The SAR results indicated that the substituent on the phenyl ring and the five-membered furan ring played key roles in the inhibition activity. Among all the furan-carboxamide compounds studied, compound $\mathbf{1 a}$ is the best one with $\mathrm{EC}_{50}$ value $1.25 \mu \mathrm{M}$ and $\mathrm{CC}_{50}$ value more than $100 \mu \mathrm{M}$, which is comparable with amantadine. Further investigation of the furan-carboxamide derivatives is underway and will be reported in due course.

\section{Experimental section}

\section{Materials and methods}

Unless otherwise noted, reagents and materials were obtained from commercial suppliers and were used without further purification. Reactions were monitored by thin layer chromatography (TLC) and column chromatography purification was performed using 230-400 mesh silica gel. NMR spectra were measured on Bruker DRX and DMX spectrometers at $400 \mathrm{MHz}$ for ${ }^{1} \mathrm{H}$ spectra and at $100 \mathrm{MHz}$ for ${ }^{13} \mathrm{C}$ spectra and calibrated from the residual solvent signal. All final products were characterized by ${ }^{1} \mathrm{H}$ NMR, ${ }^{13} \mathrm{C}$ NMR and MS analyses.

Representative procedure for synthesis of 2,5-dimethyl- $\mathrm{N}$-(2((4-nitrobenzyl)thio)-ethyl)furan-3-carboxamide 1a. To a solution of HOBT $(57.8 \mathrm{mg}, 0.428 \mathrm{mmol})$, EDCI $(82.1 \mathrm{mg}, 0.428$ $\mathrm{mmol}$ ) and 2,5-dimethylfuran-3-carboxylic acid $(60 \mathrm{mg}, 0.428$ $\mathrm{mmol}$ ) in dry DMF (2 mL) under Ar atmosphere, a solution of 2((4-nitrobenzyl)thio)ethan-1-amine $(109.0 \mathrm{mg}, 0.514 \mathrm{mmol})$ in DMF $(1 \mathrm{~mL})$ was add. The reaction solution was stirred at room temperature overnight, after completed, extracted with ethyl acetate $(3 \times 30 \mathrm{~mL})$ and brine, dried over anhydrous sodium sulphate, concentrated by vacuum pump, and purified by flash chromatography on a silica gel column, eluting with petroleum ether/ethyl acetate (gradient from $10: 1$ to $6: 1$ ) to afford $27.8 \mathrm{mg}$ (yield $19 \%)$ of 2,5 -dimethyl- $N$-(2-((4-nitrobenzyl)-thio) ethyl)-furan-3-carboxamide 1a as white solid. Mp 105-106 ${ }^{\circ} \mathrm{C}$. ${ }^{1} \mathrm{H}$ NMR $\left(400 \mathrm{MHz}, \mathrm{CDCl}_{3}\right) \delta 8.19-8.15(\mathrm{~m}, 2 \mathrm{H}), 7.51(\mathrm{~d}, J=$ $8.7 \mathrm{~Hz}, 2 \mathrm{H}), 6.72(\mathrm{~d}, J=0.8 \mathrm{~Hz}, 1 \mathrm{H}), 6.10(\mathrm{~s}, 1 \mathrm{H}), 3.82(\mathrm{~s}, 2 \mathrm{H})$, 
$3.55(\mathrm{q}, J=6.4 \mathrm{~Hz}, 2 \mathrm{H}), 2.65(\mathrm{t}, J=6.5 \mathrm{~Hz}, 2 \mathrm{H}), 2.63(\mathrm{~s}, 3 \mathrm{H}), 2.39$ $(\mathrm{s}, 3 \mathrm{H}) .{ }^{13} \mathrm{C} \mathrm{NMR}\left(100 \mathrm{MHz}, \mathrm{CDCl}_{3}\right) \delta 164.60,147.03,145.95$, $142.80,136.18,131.01,129.74$, 123.99, 123.88, 38.02, 35.24, 31.34, 15.03, 14.87. HRMS (ESI) calcd for $\mathrm{C}_{16} \mathrm{H}_{18} \mathrm{~N}_{2} \mathrm{O}_{4} \mathrm{~S}[\mathrm{M}+\mathrm{H}]^{+}$ 335.1066 , found 335.1058 .

$\mathbf{N}$-(2-(Benzylthio)ethyl)-2,5-dimethylfuran-3-carboxamide $\mathbf{1 b}$. $78 \%$ yield as colorless oil. ${ }^{1} \mathrm{H}$ NMR $\left(400 \mathrm{MHz}, \mathrm{CDCl}_{3}\right) \delta 7.32-7.27$ (m, 4H), 7.26-7.20 (m, 1H), $6.19(\mathrm{~s}, 1 \mathrm{H}), 6.00(\mathrm{~d}, J=0.7 \mathrm{~Hz}, 1 \mathrm{H})$, $3.72(\mathrm{~s}, 2 \mathrm{H}), 3.49(\mathrm{dd}, J=12.5,6.2 \mathrm{~Hz}, 2 \mathrm{H}), 2.62(\mathrm{t}, J=6.4 \mathrm{~Hz}$, $2 \mathrm{H}), 2.52(\mathrm{~s}, 3 \mathrm{H}), 2.22(\mathrm{~s}, 3 \mathrm{H}) .{ }^{13} \mathrm{C} \mathrm{NMR}\left(100 \mathrm{MHz}, \mathrm{CDCl}_{3}\right)$ $\delta 164.19,155.09,149.92,138.09,128.86,128.62,127.17,115.95$, 104.14, 37.75, 35.76, 31.22, 13.51, 13.30. HRMS (ESI) calcd for $\mathrm{C}_{16} \mathrm{H}_{19} \mathrm{NO}_{2} \mathrm{~S}[\mathrm{M}+\mathrm{H}]^{+} 290.1215$, found 290.1208.

$\mathrm{N}$-(2-((4-Fluorobenzyl)thio)ethyl)-2,5-dimethylfuran-3-carboxamide 1c. $20 \%$ yield as yellow solid. Mp 79-80 ${ }^{\circ} \mathrm{C} .{ }^{1} \mathrm{H}$ NMR $(400$ $\left.\mathrm{MHz} \mathrm{CDCl}_{3}\right) \delta$ 7.35-7.22 (m, 2H), 7.02-6.95 (m, 2H), $6.73(\mathrm{~d}, J=$ $0.9 \mathrm{~Hz}, 1 \mathrm{H}), 6.18(\mathrm{~s}, 1 \mathrm{H}), 3.71(\mathrm{~s}, 2 \mathrm{H}), 3.53(\mathrm{dd}, J=12.5,6.1 \mathrm{~Hz}$, 2H), 2.68-2.60 (m, 5H), 2.38 (s, 3H). ${ }^{13} \mathrm{C} \mathrm{NMR} \mathrm{(100} \mathrm{MHz,} \mathrm{CDCl}_{3}$ ) $\delta 164.61,142.57,136.03,133.77,133.74,131.23,130.44,130.36$, 124.15, 115.59, 115.38, 37.88, 34.98, 31.16, 15.02, 14.84. HRMS (ESI) calcd for $\mathrm{C}_{16} \mathrm{H}_{18} \mathrm{FNO}_{2} \mathrm{~S}[\mathrm{M}+\mathrm{H}]^{+} 308.1121$, found 308.1115 .

$\mathrm{N}$-(2-((4-Chlorobenzyl)thio)ethyl)-2,5-dimethylfuran-3-carboxamide 1d. $11 \%$ yield as colorless oil. ${ }^{1} \mathrm{H}$ NMR (400 $\left.\mathrm{MHz}, \mathrm{CDCl}_{3}\right)$ $\delta$ 7.31-7.21 (m, 4H), 6.07 (s, 1H), 5.98 (d, $J=0.5 \mathrm{~Hz}, 1 \mathrm{H}), 3.69$ (s, $2 \mathrm{H}), 3.51$ (dd, $J=12.4,6.3 \mathrm{~Hz}, 2 \mathrm{H}), 2.62(\mathrm{t}, J=6.4 \mathrm{~Hz}, 2 \mathrm{H}), 2.53$ (s, 3H), 2.24 (s, 3H). ${ }^{13} \mathrm{C} \mathrm{NMR}\left(100 \mathrm{MHz}, \mathrm{CDCl}_{3}\right) \delta 164.19,155.22$, 150.04, 136.60, 132.93, 130.20, 128.76, 115.79, 103.98, 37.72, 35.09, 31.19, 13.51, 13.31. HRMS (ESI) calcd for $\mathrm{C}_{16} \mathrm{H}_{18} \mathrm{ClNO}_{2} \mathrm{~S}$ $[\mathrm{M}+\mathrm{H}]^{+}$324.0825, found 324.0818.

$\mathrm{N}$-(2-((4-Bromobenzyl)thio)ethyl)-2,5-dimethylfuran-3-carboxamide 1e. $37 \%$ yield as colorless oil. ${ }^{1} \mathrm{H} \mathrm{NMR}\left(400 \mathrm{MHz}, \mathrm{CDCl}_{3}\right)$ $\delta$ 7.47-7.37 (m, 2H), 7.24-7.14 (m, 2H), 6.06 (s, 1H), 5.98 (d, $J=$ $0.6 \mathrm{~Hz}, 1 \mathrm{H}), 3.67(\mathrm{~s}, 2 \mathrm{H}), 3.51(\mathrm{dd}, J=12.5,6.2 \mathrm{~Hz}, 2 \mathrm{H}), 2.62(\mathrm{t}, J$ $=6.5 \mathrm{~Hz}, 2 \mathrm{H}), 2.53(\mathrm{~s}, 3 \mathrm{H}), 2.24(\mathrm{~s}, 3 \mathrm{H}) .{ }^{13} \mathrm{C} \mathrm{NMR}(100 \mathrm{MHz}$, $\left.\mathrm{CDCl}_{3}\right) \delta 164.20,155.19,150.02,137.15,131.70,130.56,121.00$, 115.82, 104.01, 37.77, 35.15, 31.19, 13.51, 13.31. HRMS (ESI) calcd for $\mathrm{C}_{16} \mathrm{H}_{18} \mathrm{BrNO}_{2} \mathrm{~S}[\mathrm{M}+\mathrm{H}]^{+} 370.0299$, found 370.0291 .

$\mathrm{N}$-(2-((4-Cyanobenzyl)thio)ethyl)-2,5-dimethylfuran-3-carboxamide 1f. $56 \%$ yield as colorless oil. ${ }^{1} \mathrm{H} \mathrm{NMR}\left(400 \mathrm{MHz}, \mathrm{CDCl}_{3}\right)$ $\delta 7.66-7.55(\mathrm{~m}, 2 \mathrm{H}), 7.45(\mathrm{~d}, J=8.4 \mathrm{~Hz}, 2 \mathrm{H}), 6.12(\mathrm{~s}, 1 \mathrm{H}), 5.99$ (s, $1 \mathrm{H}), 3.77(\mathrm{~s}, 2 \mathrm{H}), 3.52(\mathrm{q}, J=6.5 \mathrm{~Hz}, 2 \mathrm{H}), 2.62(\mathrm{t}, J=6.6 \mathrm{~Hz}, 2 \mathrm{H})$, $2.53(\mathrm{~s}, 3 \mathrm{H}), 2.24(\mathrm{~s}, 3 \mathrm{H}) .{ }^{13} \mathrm{C}$ NMR $\left(100 \mathrm{MHz}, \mathrm{CDCl}_{3}\right) \delta 164.24$, 155.29, 150.07, 143.90, 132.40, 129.65, 118.75, 115.73, 110.91, 103.94, 37.89, 35.54, 31.28, 13.50, 13.30. HRMS (ESI) calcd for $\mathrm{C}_{17} \mathrm{H}_{18} \mathrm{~N}_{2} \mathrm{O}_{2} \mathrm{~S}[\mathrm{M}+\mathrm{H}]^{+}$315.1167, found 315.1160.

2,5-Dimethyl- $N$-(2-((4-(trifluoromethyl)benzyl)thio)ethyl)furan3-carboxamide 1g. $35 \%$ yield as white solid. Mp $62-63{ }^{\circ} \mathrm{C} .{ }^{1} \mathrm{H}$ NMR (400 MHz, $\left.\mathrm{CDCl}_{3}\right) \delta 7.57(\mathrm{~d}, J=8.1 \mathrm{~Hz}, 2 \mathrm{H}), 7.44(\mathrm{~d}, J=$ $8.0 \mathrm{~Hz}, 2 \mathrm{H}), 6.06(\mathrm{~s}, 1 \mathrm{H}), 5.98(\mathrm{~s}, 1 \mathrm{H}), 3.77(\mathrm{~s}, 2 \mathrm{H}), 3.53$ (q, $J=$ $6.3 \mathrm{~Hz}, 2 \mathrm{H}), 2.63(\mathrm{t}, J=6.5 \mathrm{~Hz}, 2 \mathrm{H}), 2.53(\mathrm{~s}, 3 \mathrm{H}), 2.24(\mathrm{~s}, 3 \mathrm{H}) .{ }^{13} \mathrm{C}$ NMR (100 MHz, $\mathrm{CDCl}_{3}$ ) $\delta$ 164.22, 155.27, 150.07, 142.28, 129.19, 125.61, 125.58, 125.54, 125.50, 115.76, 103.93, 37.75, 35.30, 31.27, 13.48, 13.25. HRMS (ESI) calcd for $\mathrm{C}_{17} \mathrm{H}_{18} \mathrm{~F}_{3} \mathrm{NO}_{2} \mathrm{~S}[\mathrm{M}+\mathrm{H}]^{+}$ 358.1089 , found 358.1082 .

2,5-Dimethyl- $N$-(2-((4-methylbenzyl)thio)ethyl)furan-3-carboxamide 1h. $61 \%$ yield as colorless oil. ${ }^{1} \mathrm{H} \mathrm{NMR}\left(400 \mathrm{MHz}, \mathrm{CDCl}_{3}\right)$ $\delta 7.20(\mathrm{~d}, J=8.0 \mathrm{~Hz}, 2 \mathrm{H}), 7.11(\mathrm{~d}, J=7.8 \mathrm{~Hz}, 2 \mathrm{H}), 6.12(\mathrm{~s}, 1 \mathrm{H}), 5.99$ (s, 1H), $3.69(\mathrm{~s}, 2 \mathrm{H}), 3.49(\mathrm{dd}, J=12.4,6.0 \mathrm{~Hz}, 2 \mathrm{H}), 2.62(\mathrm{t}, J=$ $6.4 \mathrm{~Hz}, 2 \mathrm{H}), 2.52(\mathrm{~s}, 3 \mathrm{H}), 2.32(\mathrm{~s}, 3 \mathrm{H}), 2.24(\mathrm{~s}, 3 \mathrm{H}) .{ }^{13} \mathrm{C}$ NMR $(100$ $\left.\mathrm{MHz}, \mathrm{CDCl}_{3}\right) \delta 164.16,155.10,149.94,136.84,134.94,129.32$, 128.74, 115.92, 104.10, 37.64, 35.42, 31.18, 21.12, 13.50, 13.31. HRMS (ESI) calcd for $\mathrm{C}_{17} \mathrm{H}_{21} \mathrm{NO}_{2} \mathrm{~S}[\mathrm{M}+\mathrm{H}]^{+} 304.1371$, found 304.1364 .

$N$-(2-((4-Methoxybenzyl)thio)ethyl)-2,5-dimethylfuran-3-carboxamide 1i. $48 \%$ yield as white solid. Mp 79-81 ${ }^{\circ} \mathrm{C}$. ${ }^{1} \mathrm{H}$ NMR (400 $\left.\mathrm{MHz}, \mathrm{CDCl}_{3}\right) \delta$ 7.26-7.15 (m, 2H), 6.89-6.74 (m, 2H), $6.23(\mathrm{~s}, 1 \mathrm{H})$, $6.00(\mathrm{~s}, 1 \mathrm{H}), 3.77(\mathrm{~s}, 3 \mathrm{H}), 3.68(\mathrm{~s}, 2 \mathrm{H}), 3.49(\mathrm{q}, J=6.2 \mathrm{~Hz}, 2 \mathrm{H}), 2.61$ $(\mathrm{t}, J=6.4 \mathrm{~Hz}, 2 \mathrm{H}), 2.52(\mathrm{~s}, 3 \mathrm{H}), 2.22(\mathrm{~s}, 3 \mathrm{H}) .{ }^{13} \mathrm{C} \mathrm{NMR}(100 \mathrm{MHz}$, $\left.\mathrm{CDCl}_{3}\right) \delta 164.22,158.67,155.08,149.90,129.99,129.93,115.95$, 113.97, 104.14, 55.24, 37.81, 35.13, 31.07, 13.49, 13.27. HRMS (ESI) calcd for $\mathrm{C}_{17} \mathrm{H}_{21} \mathrm{NO}_{3} \mathrm{~S}[\mathrm{M}+\mathrm{H}]^{+} 320.1320$, found 320.1311 .

2,5-Dimethyl- $N$-(2-((2-nitrobenzyl)thio)ethyl)furan-3-carboxamide 1j. $67 \%$ yield as yellow oil. ${ }^{1} \mathrm{H}$ NMR (400 $\mathrm{MHz}, \mathrm{CDCl}_{3}$ ) $\delta 7.97(\mathrm{dd}, J=8.1,1.2 \mathrm{~Hz}, 1 \mathrm{H}), 7.56(\mathrm{~m}, 1 \mathrm{H}), 7.50-7.37(\mathrm{~m}, 2 \mathrm{H})$, $6.19(\mathrm{~s}, 1 \mathrm{H}), 6.04(\mathrm{~d}, J=0.7 \mathrm{~Hz}, 1 \mathrm{H}), 4.10(\mathrm{~s}, 2 \mathrm{H}), 3.52(\mathrm{q}, J=$ $6.2 \mathrm{~Hz}, 2 \mathrm{H}), 2.68(\mathrm{t}, J=6.4 \mathrm{~Hz}, 2 \mathrm{H}), 2.53(\mathrm{~d}, J=3.0 \mathrm{~Hz}, 3 \mathrm{H}), 2.24$ (s, 3H). ${ }^{13} \mathrm{C}$ NMR (100 MHz, $\left.\mathrm{CDCl}_{3}\right) \delta 164.21,155.19,150.00$, 148.60, 133.83, 133.24, 132.12, 128.44, 125.49, 115.81, 104.07, $37.84,33.20,32.03,13.50$, 13.30. HRMS (ESI) calcd for $\mathrm{C}_{16} \mathrm{H}_{18} \mathrm{~N}_{2} \mathrm{O}_{4} \mathrm{~S}[\mathrm{M}+\mathrm{H}]^{+}$335.1066, found 335.1058.

2,5-Dimethyl- $\mathrm{N}$-(2-((3-nitrobenzyl)thio)ethyl)furan-3-carboxamide 1k. $47 \%$ yield as white solid. Mp $113-115{ }^{\circ} \mathrm{C} .{ }^{1} \mathrm{H}$ NMR $\left(400 \mathrm{MHz}, \mathrm{CDCl}_{3}\right) \delta 8.21(\mathrm{~d}, J=1.5 \mathrm{~Hz}, 1 \mathrm{H}), 8.09(\mathrm{~d}, J=8.2 \mathrm{~Hz}$, $1 \mathrm{H}), 7.67$ (d, $J=7.5 \mathrm{~Hz}, 1 \mathrm{H}), 7.56-7.43(\mathrm{~m}, 1 \mathrm{H}), 6.28-6.13(\mathrm{~m}$, $1 \mathrm{H}), 6.01(\mathrm{~s}, 1 \mathrm{H}), 3.83(\mathrm{~s}, 2 \mathrm{H}), 3.54(\mathrm{q}, J=6.2 \mathrm{~Hz}, 2 \mathrm{H}), 2.65(\mathrm{t}, J=$ $6.4 \mathrm{~Hz}, 2 \mathrm{H}), 2.53(\mathrm{~s}, 3 \mathrm{H}), 2.23(\mathrm{~s}, 3 \mathrm{H}) .{ }^{13} \mathrm{C} \mathrm{NMR}(100 \mathrm{MHz}$, $\left.\mathrm{CDCl}_{3}\right) \delta 164.27,155.31,150.06,148.33,140.49,135.07,129.53$, 123.70, 122.22, 115.71, 103.95, 37.96, 35.23, 31.38, 13.48, 13.26. HRMS (ESI) calcd for $\mathrm{C}_{16} \mathrm{H}_{18} \mathrm{~N}_{2} \mathrm{O}_{4} \mathrm{~S}[\mathrm{M}+\mathrm{H}]^{+} 335.1066$, found 335.1057.

$\mathrm{N}$-(2-((3-Chlorobenzyl)thio)ethyl)-2,5-dimethylfuran-3-carboxamide 11. $39 \%$ yield as colorless oil. ${ }^{1} \mathrm{H}$ NMR (400 $\mathrm{MHz}, \mathrm{CDCl}_{3}$ ) $\delta 7.33(\mathrm{~s}, 1 \mathrm{H}), 7.26-7.15(\mathrm{~m}, 3 \mathrm{H}), 6.11(\mathrm{~s}, 1 \mathrm{H}), 5.99(\mathrm{~s}, 1 \mathrm{H}), 3.69(\mathrm{~s}$, $2 \mathrm{H}), 3.51$ (q, $J=6.2 \mathrm{~Hz}, 2 \mathrm{H}), 2.64(\mathrm{t}, J=6.4 \mathrm{~Hz}, 2 \mathrm{H}), 2.53(\mathrm{~d}, J=$ $2.8 \mathrm{~Hz}, 3 \mathrm{H}), 2.24(\mathrm{~s}, 3 \mathrm{H}) .{ }^{13} \mathrm{C} \mathrm{NMR}\left(100 \mathrm{MHz}, \mathrm{CDCl}_{3}\right) \delta 164.24$, $155.25,150.02$, 140.22, 134.41, 129.84, 128.90, 127.39, 127.03, 115.80, 104.01, 37.73, 35.30, 31.33, 13.51, 13.29. HRMS (ESI) calcd for $\mathrm{C}_{16} \mathrm{H}_{18} \mathrm{ClNO}_{2} \mathrm{~S}[\mathrm{M}+\mathrm{H}]^{+} 324.0825$, found 324.0818 .

$\boldsymbol{N}$-(2-((4-Chlorobenzyl)thio)ethyl)furan-3-carboxamide $\mathbf{1 m}$. $60 \%$ yield as white solid. Mp $126-128{ }^{\circ} \mathrm{C} .{ }^{1} \mathrm{H}$ NMR $(400 \mathrm{MHz}$, $\left.\mathrm{CDCl}_{3}\right) \delta 7.93(\mathrm{~s}, 1 \mathrm{H}), 7.43(\mathrm{~s}, 1 \mathrm{H}), 7.32-7.22(\mathrm{~m}, 4 \mathrm{H}), 6.61(\mathrm{~s}, 1 \mathrm{H})$, $6.39(\mathrm{~s}, 1 \mathrm{H}), 3.69(\mathrm{~s}, 2 \mathrm{H}), 3.53(\mathrm{q}, J=6.3 \mathrm{~Hz}, 2 \mathrm{H}), 2.62(\mathrm{t}, J=$ $6.5 \mathrm{~Hz}, 2 \mathrm{H}) .{ }^{13} \mathrm{C} \mathrm{NMR}\left(100 \mathrm{MHz}, \mathrm{CDCl}_{3}\right) \delta 162.72,144.84,143.85$, 136.55, 132.97, 130.20, 128.79, 122.38, 108.25, 38.03, 35.13, 31.00. HRMS (ESI) calcd for $\mathrm{C}_{14} \mathrm{H}_{14} \mathrm{ClNO}_{2} \mathrm{~S}[\mathrm{M}+\mathrm{H}]^{+}$296.0512, found 296.0505 .

$\boldsymbol{N}$-(2-((4-Chlorobenzyl)thio)ethyl)furan-2-carboxamide $\mathbf{1 n}$. $34 \%$ yield as white solid. Mp 91-93 ${ }^{\circ} \mathrm{C}$. ${ }^{1} \mathrm{H}$ NMR $(400 \mathrm{MHz}$, $\left.\mathrm{CDCl}_{3}\right) \delta 7.45(\mathrm{dd}, J=1.7,0.8 \mathrm{~Hz}, 1 \mathrm{H}), 7.31-7.24(\mathrm{~m}, 4 \mathrm{H}), 7.11$ $(\mathrm{dd}, J=3.5,0.8 \mathrm{~Hz}, 1 \mathrm{H}), 6.70(\mathrm{~s}, 1 \mathrm{H}), 6.50(\mathrm{dd}, J=3.5,1.8 \mathrm{~Hz}$, $1 \mathrm{H}), 3.72(\mathrm{~s}, 2 \mathrm{H}), 3.57(\mathrm{q}, J=6.4 \mathrm{~Hz}, 2 \mathrm{H}), 2.64(\mathrm{t}, J=6.5 \mathrm{~Hz}, 2 \mathrm{H})$. ${ }^{13} \mathrm{C} \mathrm{NMR}\left(100 \mathrm{MHz}, \mathrm{CDCl}_{3}\right) \delta 158.41,147.76,144.02,136.55$, $132.95,130.22,128.77,114.40,112.22,37.75,35.17,31.02$. 
HRMS (ESI) calcd for $\mathrm{C}_{14} \mathrm{H}_{14} \mathrm{ClNO}_{2} \mathrm{~S}[\mathrm{M}+\mathrm{H}]^{+}$296.0512, found 296.0507.

$\mathrm{N}$-(2-((4-Chlorobenzyl)thio)ethyl)-5-methylfuran-2-carboxamide 10. $55 \%$ yield as yellow oil. ${ }^{1} \mathrm{H} \mathrm{NMR}\left(400 \mathrm{MHz}, \mathrm{CDCl}_{3}\right) \delta 7.20(\mathrm{~s}$, $4 \mathrm{H}), 6.94(\mathrm{~d}, J=3.3 \mathrm{~Hz}, 1 \mathrm{H}), 6.56(\mathrm{~s}, 1 \mathrm{H}), 6.03-6.01(\mathrm{~m}, 1 \mathrm{H}), 3.64(\mathrm{~s}$, $2 \mathrm{H}), 3.49$ (q, $J=6.5 \mathrm{~Hz}, 2 \mathrm{H}), 2.56(\mathrm{t}, J=6.6 \mathrm{~Hz}, 2 \mathrm{H}), 2.27(\mathrm{~s}, 3 \mathrm{H})$. ${ }^{13} \mathrm{C}$ NMR $\left(100 \mathrm{MHz}, \mathrm{CDCl}_{3}\right) \delta 158.69,154.70,146.04,136.59$, 132.90, 130.23, 128.74, 115.79, 108.64, 37.81, 35.16, 31.05, 13.88. HRMS (ESI) calcd for $\mathrm{C}_{15} \mathrm{H}_{16} \mathrm{ClNO}_{2} \mathrm{~S}[\mathrm{M}+\mathrm{H}]^{+} 310.0669$, found 310.0662 .

$\mathrm{N}$-(2-((4-Chlorobenzyl)thio)ethyl)-2,5-dimethylthiophene-3carboxamide 1p. 29\% yield as colorless oil. ${ }^{1} \mathrm{H}$ NMR $(400 \mathrm{MHz}$, $\left.\mathrm{CDCl}_{3}\right) \delta 7.35-7.14(\mathrm{~m}, 4 \mathrm{H}), 6.72(\mathrm{~d}, J=0.9 \mathrm{~Hz}, 1 \mathrm{H}), 6.15(\mathrm{~s}$, $1 \mathrm{H}), 3.69(\mathrm{~s}, 2 \mathrm{H}), 3.52(\mathrm{dd}, J=12.5,6.1 \mathrm{~Hz}, 2 \mathrm{H}), 2.66-2.60(\mathrm{~m}$, $5 \mathrm{H}), 2.39(\mathrm{~s}, 3 \mathrm{H}) .{ }^{13} \mathrm{C} \mathrm{NMR}\left(100, \mathrm{CDCl}_{3}\right) \delta 164.62,142.59$, 136.59, 136.04, 132.95, 131.22, 130.21, 128.77, 124.15, 37.90, 35.06, 31.17, 15.04, 14.85. HRMS (ESI) calcd for $\mathrm{C}_{16} \mathrm{H}_{18} \mathrm{ClNOS}_{2}$ $[\mathrm{M}+\mathrm{H}]^{+} 340.0597$, found 340.0590 .

$\mathrm{N}$-(2-((4-Chlorobenzyl)thio)ethyl)-2-methylthiazole-4-carboxamide 1q. 61\% yield as white solid. Mp 190-191 ${ }^{\circ} \mathrm{C} .{ }^{1} \mathrm{H}$ NMR $\left(400 \mathrm{MHz}, \mathrm{DMSO}-d_{6}\right) \delta 8.74(\mathrm{t}, J=5.6 \mathrm{~Hz}, 1 \mathrm{H}), 8.17(\mathrm{~s}, 1 \mathrm{H}), 7.43-$ $7.32(\mathrm{~m}, 4 \mathrm{H}), 3.78(\mathrm{~s}, 2 \mathrm{H}), 3.44-3.37(\mathrm{~m}, 2 \mathrm{H}), 2.66(\mathrm{~s}, 3 \mathrm{H}), 2.58-$ $2.53(\mathrm{~m}, 2 \mathrm{H}) .{ }^{13} \mathrm{C}$ NMR (100 MHz, DMSO- $\left.d_{6}\right) \delta 169.50,159.84$, 142.63, 137.71, 134.82, 131.35, 130.67, 128.31, 38.67, 33.91, 29.89, 19.12. HRMS (ESI) calcd for $\mathrm{C}_{15} \mathrm{H}_{17} \mathrm{ClN}_{2} \mathrm{OS}_{2}[\mathrm{M}+\mathrm{H}]^{+}$ 341.0549 , found 341.0542 .

$\mathrm{N}$-(2-((4-Chlorobenzyl)thio)ethyl)-2-methylbenzamide 1r. $21 \%$ yield as white solid. Mp 104-105 ${ }^{\circ} \mathrm{C} .{ }^{1} \mathrm{H}$ NMR (400 MHz, $\mathrm{CDCl}_{3}$ ) $\delta 7.36-7.28(\mathrm{~m}, 2 \mathrm{H}), 7.26(\mathrm{~s}, 4 \mathrm{H}), 7.19(\mathrm{~m}, 2 \mathrm{H}), 6.22(\mathrm{~s}, 1 \mathrm{H}), 3.70(\mathrm{~s}$, $2 \mathrm{H}), 3.54(\mathrm{dd}, J=12.6,6.2 \mathrm{~Hz}, 2 \mathrm{H}), 2.63(\mathrm{t}, J=6.4 \mathrm{~Hz}, 2 \mathrm{H}), 2.41(\mathrm{~s}$, $3 \mathrm{H}) .{ }^{13} \mathrm{C}$ NMR $\left(100 \mathrm{MHz}, \mathrm{CDCl}_{3}\right) \delta 170.21,136.59,136.17,136.06$, 132.95 , 131.05, 130.25, 129.99, 128.78, 126.76, 125.77, 38.11, 35.04, 31.10, 19.85. HRMS (ESI) calcd for $\mathrm{C}_{17} \mathrm{H}_{18} \mathrm{ClNOS}[\mathrm{M}+\mathrm{H}]^{+}$ 320.0876 , found 320.0867 .

2,5-Dimethyl- $\mathrm{N}$-(2-((4-nitrobenzyl)thio)ethyl)thiophene-3carboxamide 1s. $67 \%$ yield as yellow solid. Mp $103-106{ }^{\circ} \mathrm{C}$. ${ }^{1} \mathrm{H} \mathrm{NMR}\left(400 \mathrm{MHz}, \mathrm{CDCl}_{3}\right) \delta 8.21-8.13(\mathrm{~m}, 2 \mathrm{H}), 7.50(\mathrm{~m}, 2 \mathrm{H})$, $6.04(\mathrm{~s}, 1 \mathrm{H}), 5.97(\mathrm{~s}, 1 \mathrm{H}), 3.81(\mathrm{~s}, 2 \mathrm{H}), 3.53(\mathrm{q}, J=6.4 \mathrm{~Hz}, 2 \mathrm{H})$, $2.64(\mathrm{t}, J=6.6 \mathrm{~Hz}, 2 \mathrm{H}), 2.53(\mathrm{~s}, 3 \mathrm{H}), 2.24(\mathrm{~s}, 3 \mathrm{H}) .{ }^{13} \mathrm{C} \mathrm{NMR}$ $\left(100 \mathrm{MHz}, \mathrm{CDCl}_{3}\right) \delta 164.15,155.33,150.12,147.04,145.98$, 129.71, 123.86, 115.68, 103.85, 37.90, 35.32, 31.42, 13.47, 13.26. HRMS (ESI) calcd for $\mathrm{C}_{16} \mathrm{H}_{18} \mathrm{~N}_{2} \mathrm{O}_{3} \mathrm{~S}_{2}[\mathrm{M}+\mathrm{H}]^{+} 351.0837$, found 351.0830 .

$\mathrm{N}$-(2-((4-Cyanobenzyl)thio)ethyl)-2,5-dimethylthiophene-3carboxamide 1t. $64 \%$ yield as colorless oil. ${ }^{1} \mathrm{H}$ NMR $(400 \mathrm{MHz}$, $\left.\mathrm{CDCl}_{3}\right) \delta 7.60(\mathrm{~m}, 2 \mathrm{H}), 7.45(\mathrm{~d}, J=8.2 \mathrm{~Hz}, 2 \mathrm{H}), 6.05(\mathrm{~s}, 1 \mathrm{H})$, $5.98(\mathrm{~s}, 1 \mathrm{H}), 3.77(\mathrm{~s}, 2 \mathrm{H}), 3.52(\mathrm{q}, J=6.4 \mathrm{~Hz}, 2 \mathrm{H}), 2.62(\mathrm{t}, J=$ $6.6 \mathrm{~Hz}, 2 \mathrm{H}), 2.53(\mathrm{~s}, 3 \mathrm{H}), 2.25(\mathrm{~s}, 3 \mathrm{H}) .{ }^{13} \mathrm{C} \mathrm{NMR}(100 \mathrm{MHz}$, $\left.\mathrm{CDCl}_{3}\right) \delta 164.17,155.29,150.08,143.85,132.39,129.63$, 118.69, 115.74, 110.99, 103.89, 37.87, 35.57, 31.35, 13.47, 13.27. RMS (ESI) calcd for $\mathrm{C}_{17} \mathrm{H}_{18} \mathrm{~N}_{2} \mathrm{OS}_{2}[\mathrm{M}+\mathrm{H}]^{+} 331.0939$, found 331.0932 .

2,5-Dimethyl- $\mathrm{N}$-(4-phenylbutyl)furan-3-carboxamide $1 \mathrm{u} .51 \%$ yield as colorless oil. ${ }^{1} \mathrm{H}$ NMR $\left(400 \mathrm{MHz} \mathrm{CDCl}_{3}\right) \delta 7.33-7.24(\mathrm{~m}$, 2H), $7.19(\mathrm{~m}, 3 \mathrm{H}), 6.70(\mathrm{~d}, J=0.9 \mathrm{~Hz}, 1 \mathrm{H}), 5.77$ (s, 1H), 3.39 (dd, $J$ $=12.9,6.9 \mathrm{~Hz}, 2 \mathrm{H}), 2.67-2.61(\mathrm{~m}, 5 \mathrm{H}), 2.37(\mathrm{~s}, 3 \mathrm{H}), 1.76-1.55(\mathrm{~m}$, $4 \mathrm{H}) .{ }^{13} \mathrm{C}$ NMR $\left(100 \mathrm{MHz}, \mathrm{CDCl}_{3}\right) \delta 164.73,142.13,135.96,131.60$,
128.44, 128.37, 125.85, 124.09, 39.41, 35.52, 29.35, 28.79, 15.03, 14.78. HRMS (ESI) calcd for $\mathrm{C}_{17} \mathrm{H}_{21} \mathrm{NO}_{2}[\mathrm{M}+\mathrm{H}]^{+} 272.1651$, found 272.1642 .

$\mathrm{N}$-Benzyl-2,5-dimethylfuran-3-carboxamide $1 \mathrm{v} .58 \%$ yield as white solid. Mp 109-112 ${ }^{\circ} \mathrm{C} .{ }^{1} \mathrm{H}$ NMR $\left(400 \mathrm{MHz} \mathrm{CDCl}_{3}\right) \delta 7.34-$ $7.18(\mathrm{~m}, 5 \mathrm{H}), 6.29(\mathrm{~s}, 1 \mathrm{H}), 6.02(\mathrm{~s}, 1 \mathrm{H}), 4.50(\mathrm{~d}, J=5.8 \mathrm{~Hz}, 2 \mathrm{H})$, $2.52(\mathrm{~s}, 3 \mathrm{H}), 2.20(\mathrm{~s}, 3 \mathrm{H}) .{ }^{13} \mathrm{C}$ NMR $\left(100 \mathrm{MHz}, \mathrm{CDCl}_{3}\right) \delta 164.25$, 155.38, 149.89, 138.65, 128.64, 127.71, 127.35, 115.87, 104.15, 43.22, 13.52, 13.28. HRMS (ESI) calcd for $\mathrm{C}_{14} \mathrm{H}_{15} \mathrm{NO}_{2}[\mathrm{M}+\mathrm{H}]^{+}$ 230.1181 , found 230.1175 .

\section{Plaque reduction assay (PRA)}

MDCK (Madin-Darby canine kidney) cells were purchased from the American Type Culture Collection. A confluent monolayer of MDCK cells was prepared in 6-well plates. Cells were infected with H5N1 virus at 100 PFU per well in DMEM supplemented with $0.001 \%$ DEAE-dextran, $2 \mu \mathrm{g} \mathrm{mL}{ }^{-1}$ of TPCK-treated trypsin and $0.5 \%$ agarose in the presence of various concentrations of test compounds for $40 \mathrm{~min}$ at $37{ }^{\circ} \mathrm{C}$. Medium containing $0.001 \%$ DEAE-dextran, $2 \mu \mathrm{g} \mathrm{mL}{ }^{-1}$ of TPCK-treated trypsin, $0.5 \%$ agarose and test compounds at the same concentrations was then added. After 2-3 days incubation at $37{ }^{\circ} \mathrm{C}$ in a $\mathrm{CO}_{2}$ incubator, cell monolayers were fixed with $3 \%$ formaldehyde and stained with $0.5 \%$ crystal violet, and $\mathrm{EC}_{50}$ values were counted. Amantadine was included in all experiments as the reference compound.

\section{Cytotoxicity assay}

Cytotoxicity of compounds was tested in MDCK cells by the 3(4,5-dimethylthiazol-2-yl)-2,5-diphenyltetrazolium bromide (MTT) method. Briefly, cells were seeded at a density of $2 \times 10^{4}$ per well into 96-well plates. The next day, cell medium was removed, and fresh medium containing DMEM plus test compounds at various concentrations was added to each well. After $24 \mathrm{~h}, 5 \mathrm{mg} \mathrm{mL}{ }^{-1}$ of MTT solution was added to each well and plates were incubated for $4 \mathrm{~h}$ at $37^{\circ} \mathrm{C}$ in a $\mathrm{CO}_{2}$ incubator. Successively, a solubilization solution was added to lyse cells. After $3 \mathrm{~h}$ of incubation at $37{ }^{\circ} \mathrm{C}$, absorbance was measured at $575 \mathrm{~nm}$ using an ELISA plate reader (Tecan Sunrise).

\section{Time-of-addition assay}

Influenza virus (H5N1) was gift from Prof. Congyi Zheng (Wuhan University). The influenza virus was propagated in MDCK cells. MDCK cells were seeded in 48-well plates and were infected with $\mathrm{H} 5 \mathrm{~N} 1$ virus at an MOI of 2. Compound 1a and AMD were added at the time of infection $(0 \mathrm{~h})$ and at 1, 2, 3, 4, $6 \mathrm{~h}$ and $8 \mathrm{~h}$ after infections as indicated. Viral titers were determined at $12 \mathrm{~h}$ after infection by plaque assay as described above. Compound 1a was added at a concentration of $6.2 \mu \mathrm{M}$ and AMD $(2.8 \mu \mathrm{M})$ was used as control treatment.

\section{Acknowledgements}

We are grateful to the NSFC (81573279, 81373255), Major Project of Technology Innovation Program of Hubei Province (2016ACA126), Hubei Province's Outstanding Medical 
Academic Leader Program, and Hunan Province Cooperative Innovation Center for Molecular Target New Drug Study for support of this research.

\section{References}

1 R. G. Webster, M. Peiris, H. L. Chen and Y. Guan, Emerging Infect. Dis., 2006, 12, 3-8.

2 M. V. Itzstein and R. Thomson, in Antiviral Strategies, ed. H. G. Krausslich and R. Bartenschlager, Berlin, Heidelberg, Springer-Verlag, 2009, pp. 111-154.

3 K. Das, J. Med. Chem., 2012, 55, 6263-6277.

4 H. M. Wise, A. Foeglein, J. Sun, R. M. Dalton, S. Patel, W. Howard, E. C. Anderson, W. S. Barclay and P. Digard, J. Virol., 2009, 83, 8021-8031.

5 C. Li, Z. Bu and H. Chen, Trends Biotechnol., 2014, 32, 147156.

6 J. C. De Jong, E. C. J. Claas, A. D. M. E. Osterhaus, R. G. Webster and W. L. Lim, Nature, 1997, 389, 554.

7 K. K. W. To, K. H. L. Ng, T.-L. Que, J. M. C. Chan, K.-Y. Tsang, A. K. L. Tsang, H. Chen and K.-Y. Yuen, Emerging Microbes Infect., 2012, 1, e25.

8 G. Neumann, H. Chen, G. F. Gao, Y. Shu and Y. Kawaoka, Cell Res., 2009, 20, 51-61.

9 T. M. Uyeki, Respirology, 2008, 13(Suppl 1), S2-S9.

10 L. Du, Y. Li, G. Zhao, L. Wang, P. Zou, L. Lu, Y. Zhou and S. Jiang, J. Infect. Dis., 2013, 208, 1315-1319.

11 (a) M. Imai, T. Watanabe, M. Hatta, S. C. Das, M. Ozawa, K. Shinya, G. Zhong, A. Hanson, H. Katsura, S. Watanabe, C. Li, E. Kawakami, S. Yamada, M. Kiso, Y. Suzuki, E. A. Maher, G. Neumann and Y. Kawaoka, Nature, 2012, 486, 420-428; (b) S. Herfst, E. J. A. Schrauwen, M. Linster, S. Chutinimitkul, E. de Wit, V. J. Munster, E. M. Sorrell, T. M. Bestebroer, D. F. Burke, D. J. Smith, G. F. Rimmelzwaan, A. D. M. E. Osterhaus and R. A. M. Fouchier, Science, 2012, 336, 1534; (c) W. Zhang, Y. Shi, X. Lu, Y. Shu, J. Qi and G. F. Gao, Science, 2013, 340, 1463.

12 T. L. Blundell, B. L. Sibanda, R. W. Montalvao, S. Brewerton, V. Chelliah, C. L. Worth, N. J. Harmer, O. Davies and D. Burke, Philos. Trans. R. Soc., B, 2006, 361, 413-423.
13 S. J. F. Macdonald, R. Cameron, D. A. Demaine, G. Foster, D. Gower, J. N. Hamblin, S. Hamilton, G. J. Hart, A. P. Hill, G. G. A. Inglis, B. Jin, H. J. Jones, C. B. McConnel, J. M. Breschkin, G. Mills, I. J. Owens, N. Parry, S. E. Shanahan, D. Smith, K. G. Watson, W. Wu and S. Tucker, J. Med. Chem., 2005, 48, 2964-2971.

14 J. An, D. C. W. Lee, A. H. Y. Law, C. Yang, L. Poon, A. S. Y. Lau and S. J. W. Jones, J. Med. Chem., 2009, 52, 2667-2672.

15 G. Song, S. Yang, W. Zhang, Y. Cao, P. Wang, N. Ding, Z. Zhang, Y. Guo and Y. Li, J. Med. Chem., 2009, 52, 73687371.

16 Y. Xie, D. Xu, B. Huang, X. Ma, W. Qi, F. Shi, X. Liu, Y. Zhang and W. Xu, J. Med. Chem., 2014, 57, 8445-8458.

17 K. C. Liu, J. M. Fang, J. T. Jan, T. R. Cheng, S. Y. Wang, S. T. Yang, Y. E. Cheng and C. H. Wong, J. Med. Chem., 2012, 55, 8493-8501.

18 J. W. Pan, X. Han, N. Y. Sun, H. M. Wu, D. D. Lin, P. Tien, H. B. Zhou and S. W. Wu, RSC Adv., 2015, 5, 55100-55108.

19 X. Han, N. Sun, H. Wu, D. Guo, P. Tien, C. Dong, S. W. Wu and H. B. Zhou, J. Med. Chem., 2016, 59, 2139-2150.

20 X. Han, H. M. Wu, W. Wang, C. Dong, P. Tien, S. W. Wu and H. B. Zhou, Org. Biomol. Chem., 2014, 12, 8308-8317.

21 X. Han, H. M. Wu, C. Dong, P. Tien, W. Xie, S. W. Wu and H. B. Zhou, RSC Adv., 2015, 5, 10005-10013.

22 M. H. Shaikh, D. D. Subhedar, M. Arkile, V. M. Khedkar, N. Jadhav, D. Sarkar and B. B. Shingate, Bioorg. Med. Chem. Lett., 2016, 26, 561-569.

23 W. P. Hu, G. D. Cao, J. H. Zhu, J. Z. Li and X. H. Liu, RSC Adv., 2015, 5, 82153-82158.

24 J. Tummatorn, C. Thongsornkleeb, S. Ruchirawat and T. Gettongsong, Org. Biomol. Chem., 2013, 11, 1463-1467.

25 L. Feng, K. Lv, M. Liu, S. Wang, J. Zhao, X. You, S. Li, J. Cao and H. Guo, Eur. J. Med. Chem., 2012, 55, 125-136.

26 J. Wang, C. Ma, V. Balannik, L. H. Pinto, R. A. Lamb and W. F. DeGrado, ACS Med. Chem. Lett., 2011, 2, 307-312.

27 L. H. Pinto and R. A. Lamb, J. Biol. Chem., 2006, 281, 89979000.

28 J. Wang, Y. Wu, C. Ma, G. Fiorin, J. Wang, L. H. Pinto, R. A. Lamb, M. L. Klein and W. F. DeGrado, Proc. Natl. Acad. Sci. U. S. A., 2013, 110, 1315-1320.

29 O. Trott and A. J. Olson, J. Comput. Chem., 2010, 31, 455-461. 